\title{
Flare-Control Effectiveness at Hypersonic Speeds
}

\author{
By Konstantinos KonTIS \\ Department of Mechanical, Aerospace \& Manufacturing Engineering, University of Manchester Institute \\ of Science and Technology, P.O. Box 88, Manchester, M60 1QD, UK
}

(Received August 7th, 2003)

\begin{abstract}
The effects of flare control on the aerodynamic characteristics, performance, and stability of a cylindrical body under laminar and turbulent boundary layer conditions have been studied experimentally and computationally. The experimental study has been carried out in a hypersonic gun tunnel at a Mach number of 8.2 and a Reynolds number of 158,100, based on the cylinder diameter, at flare angles $0,5,10,15,20,25$ and 30 degrees and at pitch angles of -12 to 12 deg for the $10 \mathrm{deg}$ flare case only. The surface flow was studied using the oil-dot technique. Some information regarding the shock layer was obtained from schlieren pictures. The effects of turbulence on onset of separation were also deduced from pressure measurements over the cylinder and the flare. The forces were measured with a three-component balance equipped with semiconductor strain gauges. The effects of centre of gravity (CG) location on the aerodynamic characteristics and in particular on the $C_{\mathrm{M}_{\alpha}}$ were examined. The results under turbulent conditions and zero-incidence were compared with numerical simulations performed using a 3-D time-marching Navier-Stokes code. The magnitude of the separated region, the minimum flare angle required to induce separation, and the effects of small-scale separation are detailed.
\end{abstract}

Key Words: Hypersonic Flow, Flare, Control Effectiveness

\section{Nomenclature}

(SI units unless noted otherwise)

$C$ : Chapman-Rubesin constant

$C_{\mathrm{A}}$ : axial force coefficient

$C_{\mathrm{f}}$ : skin friction coefficient

$C_{\mathrm{M}}$ : pitching moment coefficient

$C_{\mathrm{M}_{\alpha}}$ : slope of pitching moment curve $\left(=\frac{\partial C_{\mathrm{M}}}{\partial \alpha}\right.$ per rad $)$

$C_{\mathrm{N}}$ : normal force coefficient

$C_{\mathrm{N}_{\alpha}}$ : slope of normal force curve $\left(=\frac{\partial C_{\mathrm{N}}}{\partial \alpha}\right.$ per rad $)$

$d$ : diameter

$L$ : length

$M$ : Mach number

$p$ : pressure

Re: Reynolds number

$T$ : temperature

$U$ : velocity

$x$ : measured distance in $x$-direction

$y$ : measured distance in $y$-direction

Greek letters

$\alpha$ : incidence

$\beta$ : flare angle

$\delta$ : boundary layer thickness

$\chi$ : viscous interaction parameter for laminar flow subscripts $\left(=M_{\infty}^{3} \sqrt{C / R e_{x}}\right)$

$\infty$ : freestream

cyl: cylinder

incip: incipient

L: laminar conditions

(C) 2004 The Japan Society for Aeronautical and Space Sciences sep: separation

re: reattachment

\section{Introduction}

Boundary layer separation is of interest in many phases of aerodynamics. The presence of separation causes sudden changes in pressure and heat transfer distributions and aerodynamic forces. When separation occurs, the designer must be able to judge the acceptability and desirability of the separated boundary layer by knowing the flow characteristics, i.e. extent of separation, pressure distribution, heat transfer and boundary layer steadiness. Excessive thermal loading can result in ablation, thus dynamically modifying the geometry of the projectile in flight or result in other detrimental phenomena such as amplified infra-red signatures, damage to guidance systems, etc. Separation of supersonic and hypersonic flow has been widely researched, yet recent results suggest that the aerodynamic community does not yet fully understand the mechanism involved. With the advancement of modern experimental techniques, high-resolution results are being obtained, which are suggesting that very small regions of separated flow may be occurring for small angles of flow deflections. ${ }^{1-4)}$ For very small regions of separated flow it is further suggested that effects may be seen in heat transfer, skin friction, etc. (i.e. factors governed by 'near-wall' flow), but not in parameters such as surface pressure distribution which are more commonly examined by experimental aerodynamicists.

The cylinder-flare configuration has many advantages as a space vehicle and missile: The nose shape can be adjusted to suit different thermal protection materials and to produce the required drag for a given trajectory; the centerbody 
length can be adjusted for payload size; and the flare angle and length can be varied to produce a stable configuration. The present investigation aims: a) to complement the existing database on boundary layer separation for three dimensional bodies; b) to collect the previous information and draw attention to the main trends; and c) to determine the effects of centre of gravity (CG) location on the aerodynamic characteristics and in particular $C_{\mathrm{M}_{\alpha}}$ relative to the force balance moment gauge centre and to the free-flight model $\mathrm{CG}^{5 \text { ) }}$

\section{Incipient Separation Interactions}

Experiments by Coleman and Stollery, ${ }^{6)}$ Kuehn, ${ }^{7-9)}$ Gray $^{10)}$ and Ginoux ${ }^{11)}$ have emphasised the close similarity between axisymmetric and two-dimensional separated flows. This similarity suggests that criteria derived from the large amount of available two-dimensional data may be relevant to the analysis of axisymmetric interactions.

\subsection{Laminar interactions}

Experimental studies by Needham and Stollery ${ }^{12)}$ of incipient separation in hypersonic flow have shown that for a sharp leading edge flat plate of length $L$ with a trailing edge flap at angle $\beta$ :

$$
M_{\infty} \beta_{\text {incip }}=80 \bar{\chi}_{\infty \mathrm{L}}^{1 / 2}
$$

Inger ${ }^{13)}$ has shown, using a triple deck formulation of the incipient separation interaction region, that this experimental correlation has a sound theoretical basis.

\subsection{Turbulent interactions}

$\mathrm{Kuehn}^{8)}$ has shown that the qualitative effects of geometry and test conditions on the occurrence of turbulent separation are very similar to those found for laminar boundary layers. However, it is now well established that in a wellseparated turbulent flow there is an increase in heat transfer in the separated region in contrast to the laminar case in which the heat transfer is reduced in the separated region. This is one of the most significant differences between laminar and turbulent flow. Various researchers ${ }^{1,8,14-16)}$ have found that very high incipient separation angles $\left(\cong 30^{\circ}\right)$ are possible in hypersonic flow under turbulent conditions. A collection of incipient separation data is shown in Fig. 1. The correlation and explanation of all the data pre-

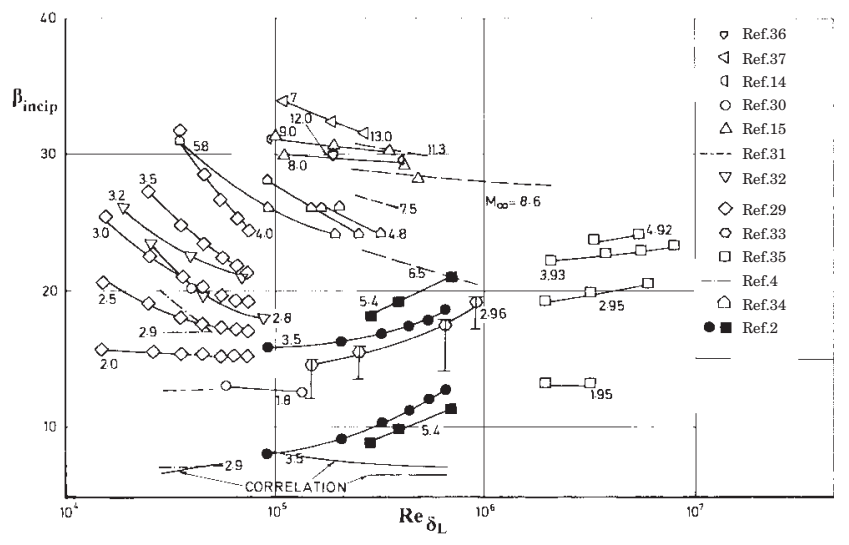

Fig. 1. Incipient separation at a wedge compression corner. sented is impossible because of the difficulty in defining the incipient separation condition in turbulent flow. Since turbulent separated flow is unsteady, most measurements in Fig. 1 reflect a mean value of $\beta_{\text {incip }}$, which varies with the technique chosen to detect incipient separation. The same figure shows the trend reversal of $\beta_{\text {incip }}$ with $\operatorname{Re}_{\delta_{\mathrm{L}}}$ at high values of Reynolds number. Stollery and Bates ${ }^{17)}$ have suggested that by analogy to the laminar case:

$$
M_{\infty}^{2} \beta_{\text {incip }}^{2} \sim\left(\frac{M_{\infty}{ }^{9} C}{R e_{\mathrm{L}}}\right)^{2 / 7}
$$

However, the experimental data described in Fig. 1 shows that the real picture is far more complex but many experiments show $\beta_{\text {incip }}$ increasing with $M_{\infty}$.

\section{Experimental Setup}

The experiments were conducted in a hypersonic gun tunnel, with air as the freestream gas, at $M_{\infty}=8.2$ and $R e_{\infty} / m=9.3 \times 10^{6}$. Descriptions of the setup and calibration of the facility are given by Stollery et al., ${ }^{18)}$ Needham ${ }^{19)}$ and Kontis. ${ }^{20,21)}$ The flow is uniform inside the test section except on the centerline, ${ }^{22)}$ where the Mach number varies by $\pm 4.5 \%$. Pressure measurements were carried out using Kulite XCS-190 absolute pressure transducers with an estimated error of $\pm 3 \%$. Discrete dots of an oil mixture were applied on the matt black painted surface of the models. The forces were measured with a three-component balance having an accuracy of normal force and pitching moment $\pm 1.5 \%$ and of axial force $\pm 2.5 \% .^{22}$ A repeatability of $97 \%$, of the force and moment coefficients, was achieved in the present investigation over six runs.

The general geometrical characteristics of the configurations used are shown in Fig. 2. Pressure models were equipped with 32 tappings along the cylinder-flare. Vortex generators were used to trip the boundary layer (placed at the nose-cylinder junction) and were based on the design developed by Coleman. ${ }^{23)}$ They consisted of a single row of delta shaped vortex generators inclined at about $30^{\circ}$ to the flow direction, $0.001 \mathrm{~m}$ high and $0.0035 \mathrm{~m}$ apart. Vortex generators increase the thickness of the boundary and produce strong vortices, which travel downstream on the cylinder, up to approximately $30 \%$ of the cylinder length. ${ }^{21)}$ Eventu-

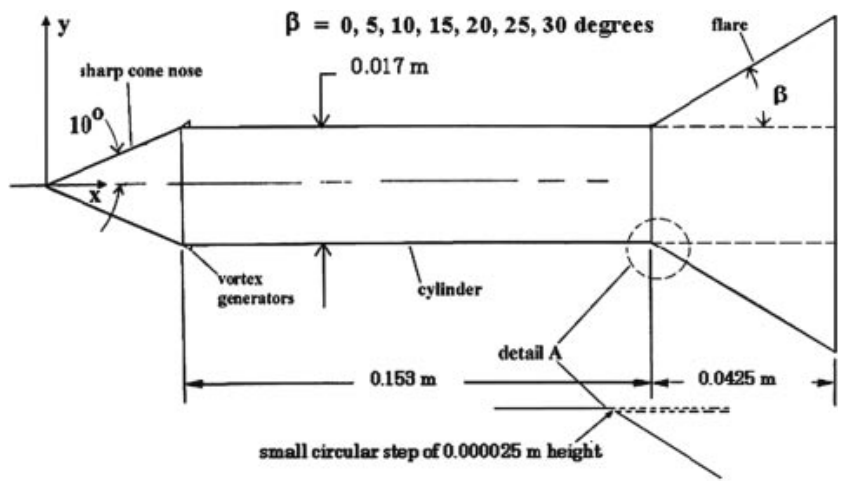

Fig. 2. Geometries of the configurations used. 
Table 1. Centre of gravity positions for models A, B and C.

CG (balance moment gauge) positions from flare base

Model A $0.0225 \mathrm{~m}$

Model B $\quad 0.0425 \mathrm{~m}$

Model C $0.0625 \mathrm{~m}$

ally these strong vortices breakdown due to surface skin friction, and reduction of their strength by turbulent dissipation of kinetic energy. Certainly, the development of the turbulent boundary layer due to forced transition is not the same as that which would be expected if the transition was natural. A naturally turbulent boundary layer does not develop strong vortices and its thickness is smaller than that of a forced turbulent boundary layer. ${ }^{21)}$

A model with a $10 \mathrm{deg}$ trailing edge flare was employed for the forces and moment measurement studies. Three models having the same geometry but different locations of centre of gravity (CG) with respect to the base of the flare were used (Table 1).

An upward (normal to the velocity vector) normal force, backward (parallel to the velocity vector) axial force and nose-up pitching moment were taken as positive. The calculated forces were non-dimensionalized using the free-stream dynamic head and the cylinder cross-section area. The pitching moments were measured at the moment reference point of each model along the body axis, measured from the base of the flare and then calculated relative to the $\mathrm{CG}$ of the free flight model located $0.05 \mathrm{~m}$ from the nose tip. The pitching moment values were non-dimensionalised using the freestream dynamic head, the cylinder cross-section area and the cylinder diameter.

\section{CFD Code, Grid Generation and Validation}

The numerical study employed a three-dimensional, high resolution, iterative, finite volume time marching NavierStokes (NS) solver. ${ }^{24,25)}$ The region of interest was discretized into small but finite hexahedral cells. The numerical integration procedure produced cell-averaged flow properties, which were assigned to the centres of each face of the hexahedral cells. The Baldwin-Lomax algebraic turbulence model $^{26)}$ was employed. It has been shown by References 24) and 25), that this turbulence model can successfully calculate separated flows with much more complicated grid geometry than that of the present study. The system of the governing equations has been solved using an explicit Harten-Yee Non-MUSCL (Monotone Upstream-centered Schemes for Conservation Laws) Modified-flux type TVD (Total Variation Diminishing) scheme, ${ }^{27)}$ accurate in time and space. Roe's average Riemann Solver was used, ${ }^{27)}$ due to its simplicity and its ability to return to the exact solution whenever the variables lie on a shock or contact discontinuity. The Courant number CFL was chosen as 0.7 to obtain rapid convergence and avoid unsteadiness in calculation. References 24) and 25) provide a detailed description of the solver. The 3-D NS solver has been validated ${ }^{24)}$ for a missile configuration tested experimentally at a Mach number of 8.2. The flowfield was simulated, assuming a perfect gas and a turbulent boundary layer over the full length of the sharp cone nose cylinder body for zero-incidence only and flare angles $0,5,10,15,20,25$ and $30 \mathrm{deg}$. The boundary conditions were non-slip and isothermal. The solution history was monitored and the steady-state solution was selected if the change of the flowfield parameters residuals became small or the maximum number of iterations was satisfied. The convergence criterion required that the residuals be smaller than $10^{-5}$ for the mass, momentum and energy equations.

The experimental data of the present study together with the boundary layer traverse data for total pressure, static pressure and hence Mach number of Ref. 1), were used to produce an appropriately validated inflow profile. Using this inflow profile to simulate the flow over the cylinder alone produces a perturbation-free skin friction coefficient and hence a perturbation-free 'near-wall' flow. ${ }^{1)}$ The grid was generated using a three-dimensional transfinite interpolation technique, ${ }^{28)}$ i.e.,

1) Generation of the one-dimensional line grids at all junctions of the domain surfaces with high density at all walls and in the region of the cylinder-flare junction, where high gradients exist, to ensure the accuracy of the simulations.

2) Generation of the two-dimensional surface grids for all of the domain boundary surfaces using the one-dimensional line grids as the interpolation boundaries and applying any geometric constraints.

3) Generation of the three-dimensional field grid by interpolation of the surface grids and ensuring the orthogonality of the grid at the wall by specifying the derivative information at the wall occurred. The cluster of the grid at the wall was controlled by either the magnitude of the derivative or the stretch factor in the interpolation. ${ }^{24)}$

4) A three-dimensional elliptic smoother was used to smooth the grid in regions where this was critical, i.e., around the sharp corners of the cylinder-flare junction. The grid points generated were the vertices of the hexahedral cells in the finite volume formulation. A secondary grid was created by the central points of the cells for the presentation of the results from the finite volume solution.

The sensitivity of the numerical solution to the computational grid was checked using five grid densities and distributions. Smoothing and stabilizing parameters were kept to a minimum during the grid-sensitivity studies. The grid was refined in each direction of the three dimensions while holding the other two dimensions fixed. The study showed a difference of less than $1 \%$ between results for grid systems of more than 65 nodes in the longitudinal direction, 65 nodes in the vertical direction and 150 nodes in the circumferential directions. A grid system of $70 \times 70 \times 155$ nodes was selected for these simulations. The minimum grid spacing was $10 \mu \mathrm{m}$ (in the neighborhood of the wall), of a typical 
value of $y^{+}<1$ for the first cell off the body surface for better resolution of the viscous layer. The mesh was extended $80 \mathrm{~mm}$ upstream of the cylinder-flare junction to ensure that the inflow boundary condition was not affecting the formation or behaviour of the separated region.

\section{Results and Discussion}

References 1) and 21) explain in detail the flowfield around the sharp cone-cylinder body. The expansion fan at the cone-cylinder junction interacts with the nose shock. The point at which influence is felt can be identified as the position at which the straight conical shock begins to curve. The flow over-expansion around the junction results in a shoulder pressure less than the free-stream. ${ }^{21}$ )

\subsection{Laminar boundary layer studies}

An adverse pressure gradient is created due to the presence of a flare in the inviscid flowfield. This pressure rise feeds upstream via the subsonic layer, which thickens the subsonic layer some distance upstream of the flare. Within the supersonic portion of the boundary layer, compression fans are generated. These fans induce a pressure rise, which thickens the subsonic layer further. This mutual interaction process between the pressure rise and the thickening boundary layer continues until equilibrium is reached. The compression waves coalesce rapidly and ultimately, within the boundary layer. ${ }^{21)}$ The present study encountered attached flow-type interaction over the sharp cone-cylinder body with a $\beta=5^{\circ}$ under laminar boundary layer conditions.

At a certain flare deflection angle the adverse pressure gradient reduces the wall velocity gradient and hence the wall skin friction to zero at the cylinder flare junction (incipient separation angle). Increasing the flare deflection angle beyond the incipient separation angle causes the flow to separate with separation and reattachment points upstream and downstream of the junction. Both the separation and reattachment points are characterised by local shockwaves, e.g. $\beta=20^{\circ}$, Fig. 3. This dual shock interaction structure differentiates separated flows from the single shock structure of attached flows. It is difficult to see the $10^{\circ}$ flare separation, because the separation region is very small and the flare angle is close to incipient separation angle. However, the $10^{\circ}$ flare separation was detected by the oil-dot technique and pressure distribution; see, for example, Fig. 4. Therefore promotion of separation is expected between $5^{\circ}$ and $10^{\circ}$ flare angles. For example, Fig. 3 shows that further increasing the flare deflection angle results in an upstream movement in the location of separation and a small downstream movement of the reattachment point. This behaviour of reattachment is an indication that the boundary layer over the flares, at all deflection angles tested, is laminar. It is believed that due to the unstable nature of the separated shear layer, transition is occurring well downstream of the interaction region.

Under the present test conditions, the equivalent incipient separation flap deflection for a sharp leading edge flat plate, as deduced by the Needham and Stollery ${ }^{12)}$ criterion (see
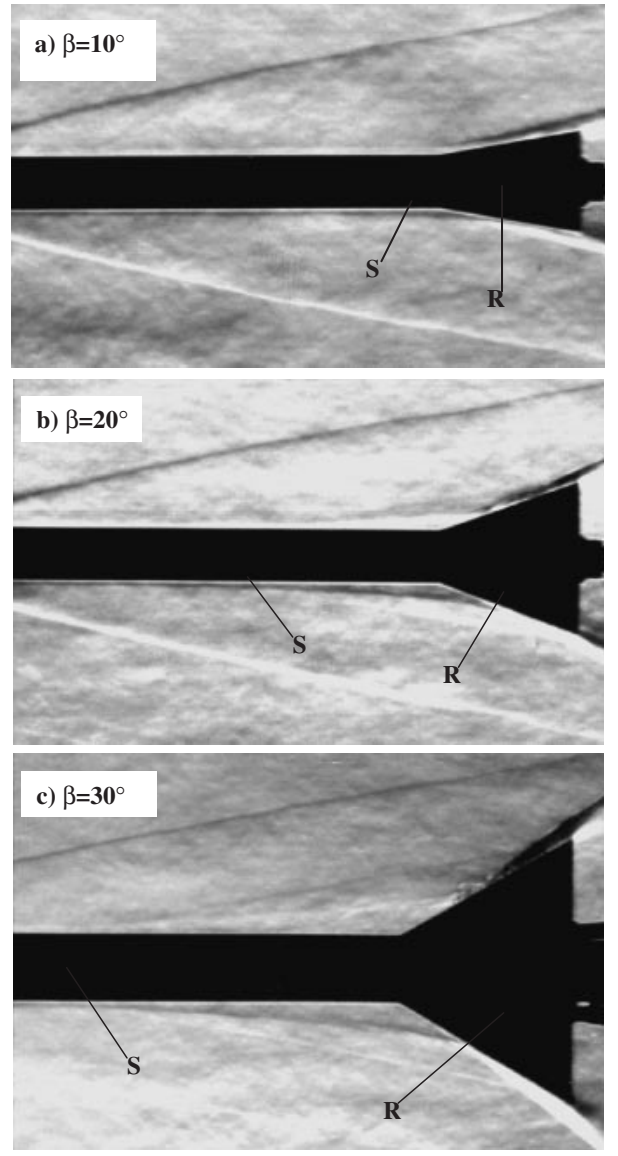

Fig. 3. The effect of flare deflection on the location of separation and reattachment in laminar flows $\left(M_{\infty}=8.2, R e_{\infty} / \mathrm{m}=9.3 \times 10^{6}, L_{\mathrm{cyl}}=\right.$ $0.153 \mathrm{~m}$, sharp cone nose).

S: separation, R: reattachment.

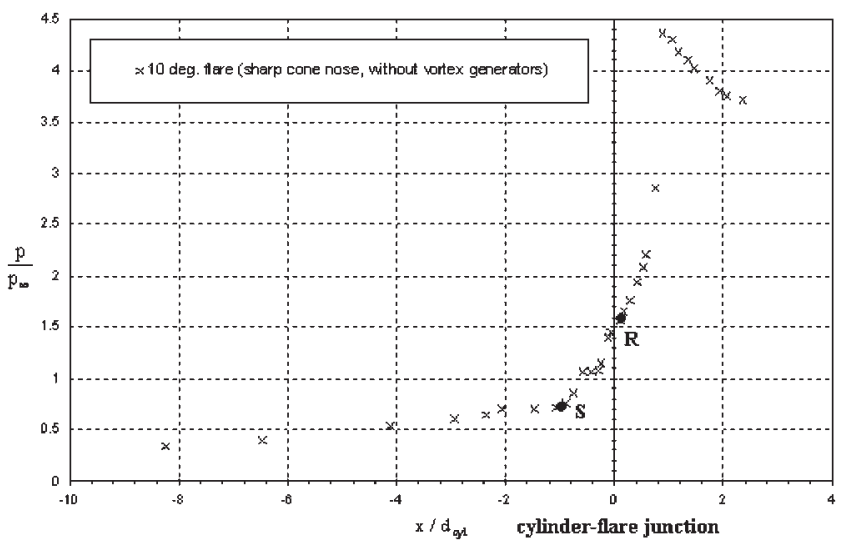

Fig. 4. Pressure distribution under laminar boundary-layer conditions for the $10^{\circ}$ flare case $\left(M_{\infty}=8.2, R e_{\infty} / \mathrm{m}=9.3 \times 10^{6}\right)$.

$\mathrm{S}$ : separation, R: reattachment.

Eq. (1)), assuming $C \cong 1$, is $6.2^{\circ}$. The lengths of the separated shear layer were measured from schlieren pictures, pressure measurements and oil-dot visualization results, and they were plotted versus the flare angle. This method indicates a value of incipient separation for the sharp conecylinder flare body of approximately $8.0^{\circ}$, which is higher than the predicted two-dimensional value of $6.2^{\circ}$, which is due to the three-dimensional relief effect associated with ax- 

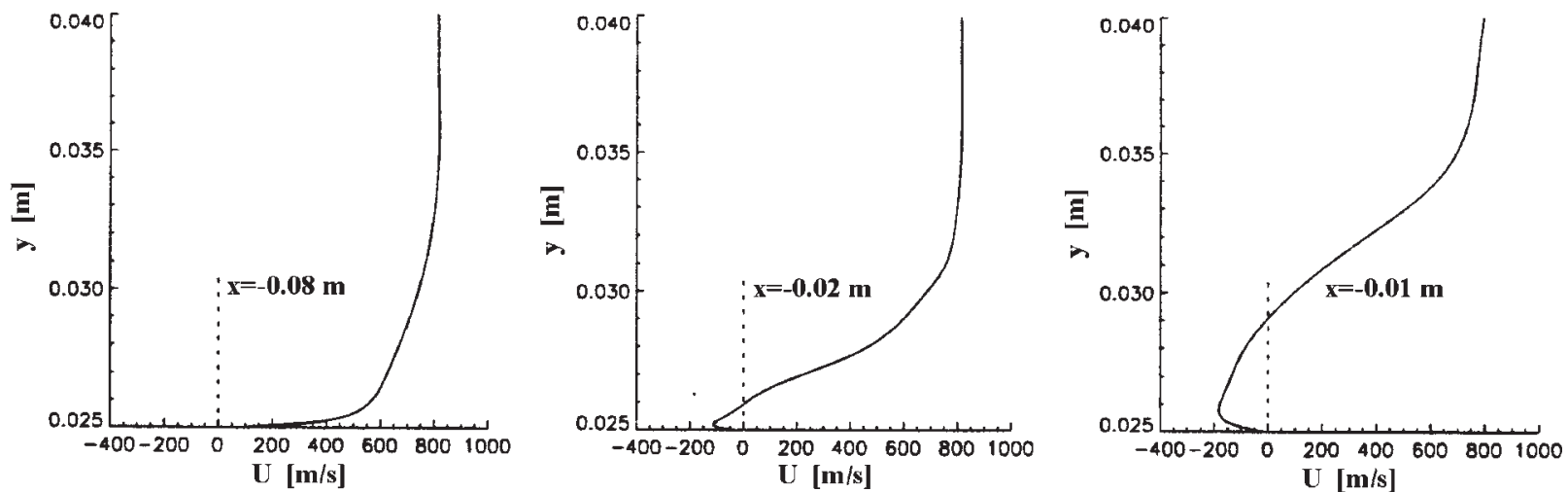

Fig. 5. Boundary layer profiles $\left(M_{\infty}=8.2, R e_{\infty} / \mathrm{m}=9.3 \times 10^{6}, \beta=30^{\circ}\right.$, computational).

Table 2. Effect of flare angle on the magnitude and location of the separated region.

\begin{tabular}{rcccc}
\hline$\beta(\mathrm{deg})$ & $\begin{array}{c}x_{\text {sep }}(\mathrm{m}) \\
\text { computation }\end{array}$ & $\begin{array}{c}x_{\text {re }}(\mathrm{m}) \\
\text { computation }\end{array}$ & $\begin{array}{c}L_{\text {sep }}(\mathrm{mm}) \\
\text { computation }\end{array}$ & $\begin{array}{c}L_{\text {sep }}(\mathrm{mm}) \\
\text { experiment }\end{array}$ \\
\hline 0 & 0 & 0 & 0 & 0 \\
5 & 0 & 0 & 0 & 0 \\
10 & $-0.25 \times 10^{-3}$ & $0.10 \times 10^{-3}$ & 0.35 & 0 \\
15 & -0.0011 & 0.0015 & 6.1 & 0 \\
20 & -0.0028 & 0.0021 & 17.8 & 4 \\
25 & -0.0065 & 0.0105 & 37.4 & 17 \\
30 & -0.0132 & 0.0168 & 67.5 & 31 \\
\hline
\end{tabular}

isymmetric bodies. The various techniques used for separation lengths detection agree reasonably well with each other within a variation range $\pm 1.5 \%$.

\subsection{Turbulent boundary layer studies}

Figure 5 shows the computational boundary layer profiles upstream of the cylinder-flare junction for the $30^{\circ}$ flare case, which shows evidence of a locally reversed flow at the junction. The latter result is also supported by the schlieren, pressure measurements and oil-dot results; see, for example, Figs. 6 and 7. The oil-dot result for the $20^{\circ}$ flare case shows that the oil-dots very near the junction of the cylinder-flare have not moved, Fig. 6. If only the oil-dot technique was available for incipient separation detection the conclusion would have been that the $20^{\circ}$ flare case shows some evidence of flow separation. However, the schlieren pictures and pressure measurements do not support this deduction; see, for example, Fig. 7. A possible explanation of the non-movement of the oil-dots near the juncture of the $20^{\circ}$ flare case is that the oil-dot technique is skin-friction dependent and its response is insensitive to very small skinfriction values. Alternatively, a very small separation bubble at the junction could exist. The latter hypothesis is supported by the computational results shown in Fig. 8, which suggest that flow has already separated at $15^{\circ}$ flare case. Table 2 summarises and compares the effect of flare angle on the magnitude and location of the separated region (obtained from the computational skin friction coefficient distribution and the experimental pressure distribution and/or oil-dot visualization). Such small-scale separations were observed by Babinsky and Edwards, ${ }^{2)}$ Appels and Richards, ${ }^{3)}$ and

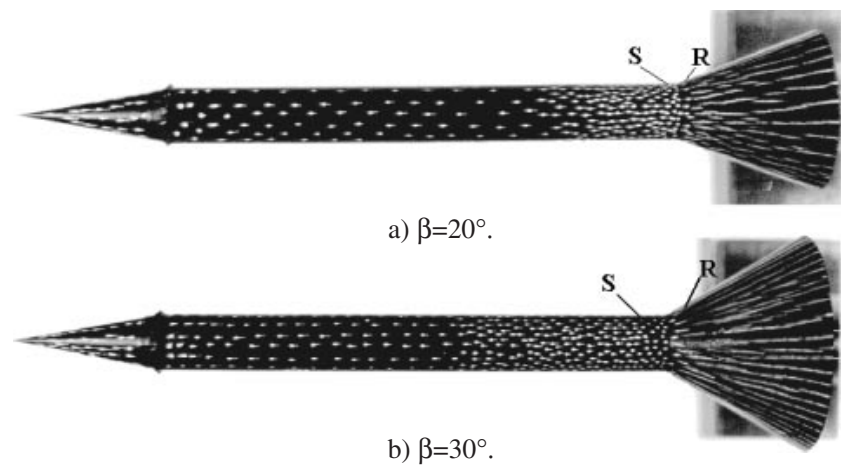

Fig. 6. Oil-dot flow visualization under turbulent boundary layer conditions $\left(M_{\infty}=8.2, R e_{\infty} / \mathrm{m}=9.3 \times 10^{6}\right)$

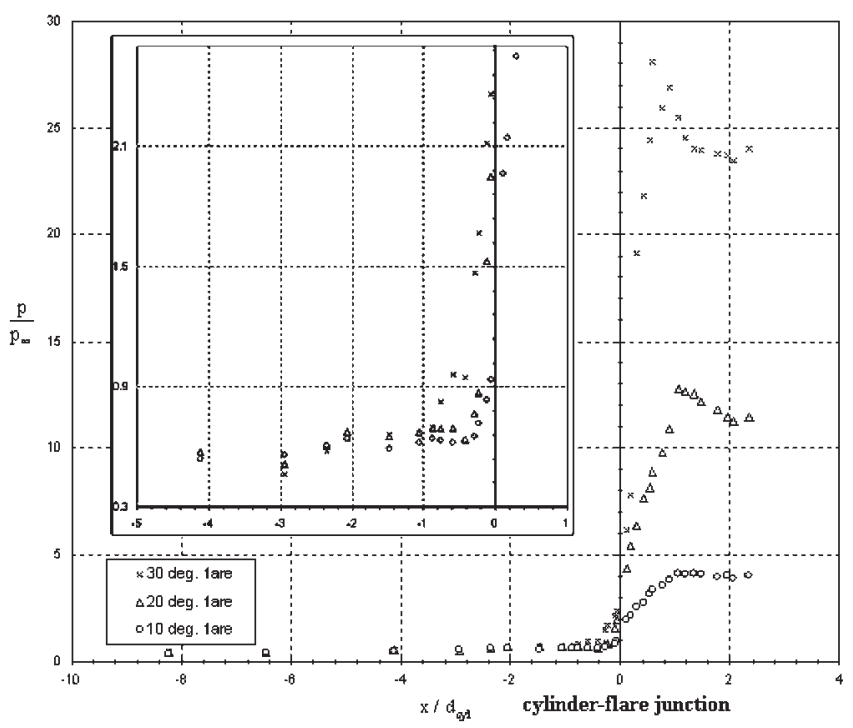

Fig. 7. Composite of pressure distributions $\left(M_{\infty}=8.2, R e_{\infty} / \mathrm{m}=9.3 \times\right.$ $10^{6}$, turbulent boundary layer).

Spaid and Frishett ${ }^{4)}$ from experiments at low corner deflection angles. The accuracy of each method used for incipient separation depends on the smallest size of separated region that can be detected. The important criterion for design purposes is when separation makes significant changes to the pressure and heat transfer distributions. That is the definition of "incipient" used here.

A pressure overshoot of increasing magnitude forms on 


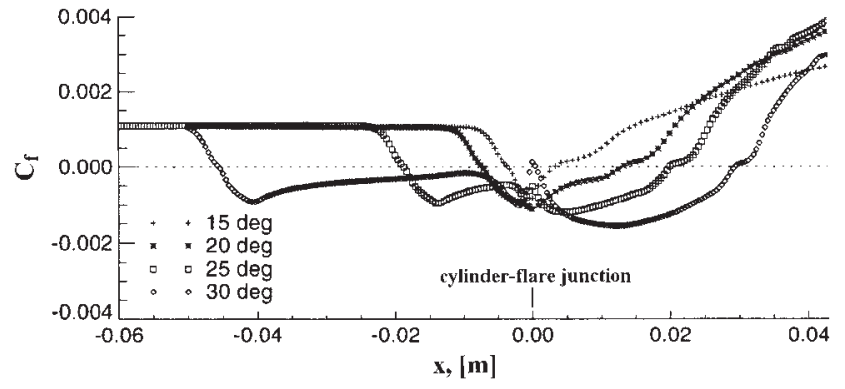

Fig. 8. Variation of skin friction distribution with flare angle.

the flare, Fig. 7. For attached flows, this is due to the locally two-dimensional pressure rise at the corner followed by a gradual decay to the cone value further downstream on the flare. For separated flows, this overshoot is due to the intersection of the separation and reattachment shocks and this results in a single shock together with a reflected expansion fan, which brings the high pressure back towards the inviscid conical pressure value. ${ }^{1)}$ The comparison of the pressure distributions for the laminar and turbulence configurations indicates that turbulence delays separation (and completely suppresses separation for the $10^{\circ}$ flare case, see Figs. 4 and 7). The delay in separation is because turbulent boundary layers are more energetic and hence more resistant to adverse pressure gradients. Incipient separation was determined by plotting the length of separated flow against flare angle and extrapolating to zero separation length. Assuming that the oil-dot result for the $20^{\circ}$ flare case is true, the experimental value of incipient separation is approximately $18^{\circ}$, and the computational value of incipient separation is approximately $13^{\circ}$. This contradicts the findings of Kuehn, ${ }^{8)}$ Coleman $^{14,16)}$ and Elfstrom ${ }^{15)}$ who found higher incipient separation angles. This is because the determination of incipient separation is very sensitive to the detection method employed. The initial state of the turbulent boundary layer is also important. Kuehn and Coleman had forced transition in their experiments while Elfstrom had free transition.

\subsection{Force measurements}

References 1) and 2) have shown that boundary-layer separation effects on flare stabilised bodies are usually manifested by a reduction in axial force at $\alpha=0^{\circ}$ and by an increase in the slopes of the normal-force and negative pitching-moment curves near zero incidence. The effect of boundary-layer separation on the windward meridian usually disappears as incidence increases and the coefficient curves become coincident or least parallel with those curves occurring for configurations free of separation over the whole incidence range.

The effect of incidence is to decrease the local Mach number and increase the local Reynolds number along the windward ray. On the leeward ray, the local Mach number increases and the local Reynolds number decreases. ${ }^{20,21,24)}$ A progressive decrease in cylinder pressure along the leeward meridian with incidence was measured which effectively increases the local flare pressure ratio and causes promotion of separation along the leeward meridian. Increasing incidence promotes the establishment of a disturbed flow region over the leeward surface of the cylinder body. This is probably, due to separation of the crossflow, resulting in the establishment of a pair of counter-rotating vortices, over the leeward region of the body. At these incidences, the flare is merged into a complex separated crossflow structure. The flow separation due to the flare has a three-dimensional structure and resembles a jet flow field interaction region for a sharp cone with hypersonic freestream.
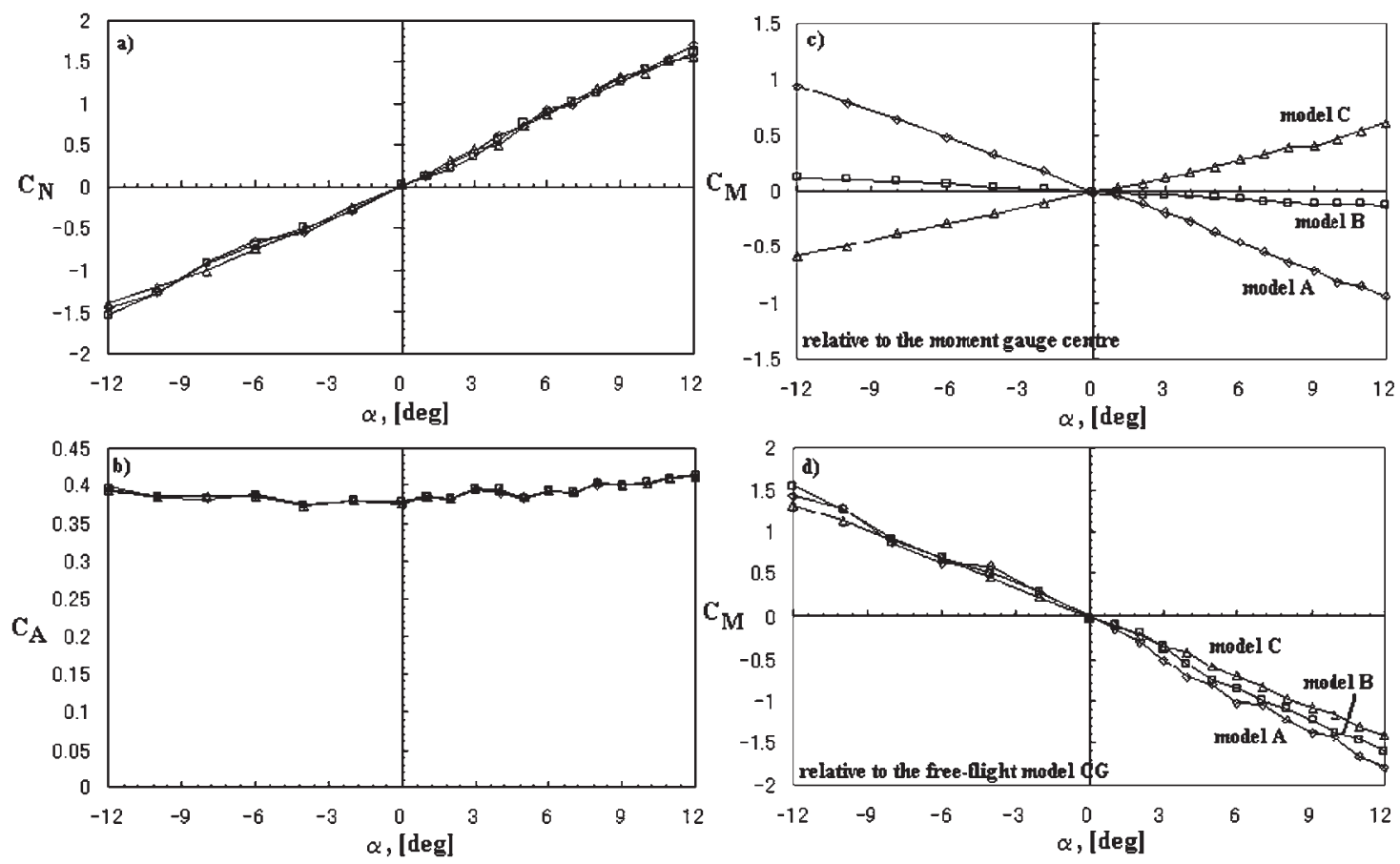

Fig. 9. Variation of the aerodynamic coefficients with incidence $\left(\beta=10^{\circ}, M_{\infty}=8.2, R e_{\infty} / \mathrm{m}=9.3 \times 10^{6}\right)$. 
Figure 9 shows the variation of the aerodynamic coefficients with incidence for models $\mathrm{A}, \mathrm{B}$ and $\mathrm{C}$. The scatter in the normal and axial force coefficients is very small. The normal force coefficient varies linearly with incidence, Fig. 9a. The pitching moment coefficient relative to the freeflight model CG shows a large scatter in the results from the three test cases, especially at the positive angles of attack, Fig. 9d. The scatter is due to slight flow non-uniformity at the test section at incidences less than $-7 \mathrm{deg}$ and over $2 \mathrm{deg}$. Reference 21) provides a comprehensive survey of the test section flow quality, and explains in detail the flow field characteristics. At incidences above $4 \mathrm{deg}$, the slopes of the three cases change abruptly. At the negative incidences to $-8 \mathrm{deg}$, the scatter in the $C_{\mathrm{M}}$ results is much lower and repeatable. The centre of pressure was at approximately 6 diameters from the nose of the body. The slopes of $C_{\mathrm{N}}$ and $C_{\mathrm{M}}$ were calculated between -6 and $6 \mathrm{deg}$ for the three models and averaged. The $C_{\mathrm{N}_{\alpha}}$ and $C_{\mathrm{M}_{\alpha}}$ are 7.78 and -7.63 respectively. Figure $9 \mathrm{c}$ shows that the slope of $C_{\mathrm{M}}$ relative to the moment gauge centre varies from positive to negative depending on whether CG position is moving towards or away from the nose; however, the slope of $C_{\mathrm{M}}$ relative to the free flight model $\mathrm{CG}$ is constant for the three models tested.

\section{Conclusions}

Flare-induced separation has been predicted at lower angles for the sharp-cone-cylinder-flare model under turbulent conditions than would have been expected on the basis of experimental work prior to that of Kontis and Stollery ${ }^{1)}$ and Babinsky and Edwards. ${ }^{2)}$ This is believed to be due to the separated region being too thin to be observed using traditional experimental techniques such as schlieren photography or surface pressure distribution analysis. This separated region has been observed using experimental techniques such as oil-dot visualisation, liquid crystal thermography or a shear-sensitive liquid crystal technique. However, the presence of separated regions exclusively within the viscous sub-layer is only detectable through skin friction coefficient and heat transfer.

Small-scale separations consistent with those observed in the present experimental study and by other researchers have been predicted computationally. Both experiment and computation indicated that the separation length increases rapidly as the flare angle is increased above $20 \mathrm{deg}$. That rapid increase in the separation length is associated with a sudden increase in the height of the separated region, which influences the external flow. That sudden increase in separation length and height corresponds to the onset of 'tranditional' separation for which perturbations to the surface pressure may be used to locate separation and reattachment. The computed size of the separated region for the $20 \mathrm{deg}$ flare case is larger than that obtained experimentally. It is conjectured that this is probably due to inadequacies in the Baldwin-Lomax turbulence model for predicting these particular flows and due to fact that the experimental and calculated flow fields are not the same, because trip was placed at the nose-cylinder junction in the experimental tests, whereas in CFD calculations, a turbulent boundary layer over the full length of the sharp cone nose cylinder body with an appropriately validated inflow profile was assumed. The normal and axial force coefficients variation with incidence is independent from the model CG position. However, the slope of $C_{\mathrm{M}}$ relative to the moment gauge centre varies from positive to negative depending on whether CG position is moving towards or away from the nose. The slope of $C_{\mathrm{M}}$ relative to the free flight model CG is constant for the three model cases tested.

\section{References}

1) Kontis, K. and Stollery, J. L.: Incipient Separation on Flared Bodies at Hypersonic Speeds, Aeronaut. J., 103 (1999), pp. 405-414.

2) Babinsky, H. and Edwards, J. A.: On the Incipient Separation of a Turbulent Hypersonic Boundary Layer, Aeronaut. J., 100 (1996), pp. 209-214.

3) Appels, C. and Richards, B. E.: Incipient Separation of a Compression Turbulent Boundary Layer, AGARD CP-168, 1975.

4) Spaid, F. W. and Frishett, J. L.: Incipient Separation of a Supersonic, Turbulent Boundary Layer, Including Effect of Heat Transfer, AIAA J., 10 (1972), pp. 915-922.

5) Fournier, E. Y., Depuis, A. D. and Edwards, J. A.: Base Cavity Effects on the Aerodynamic Characteristics of a Hypersonic Flared Projectile, J. Spacecraft Rockets, 34 (1997), pp. 737-744.

6) Coleman, G. T. and Stollery, J. L.: Incipient Separation of Axially Symmetric Hypersonic Turbulent Boundary Layers, AIAA J., 12 (1974), pp. 119-120.

7) Kuehn, D. M.: Laminar Boundary-Layer Separation Induced by Flares on Cylinders with Highly Cooled Boundary Layers at a Mach Number of 15, NASA TN D-2610, 1965.

8) Kuehn, D. M.: Turbulent Boundary-Layer Separation Induced by Flares on Cylinders at Zero Angle of Attack, NASA TR-R-117, 1961.

9) Kuehn, D. M.: Laminar Boundary-Layer Separation Induced by Flares on Cylinders at Zero Angle of Attack, NASA TR-R-146, 1962.

10) Gray, J.: Investigation of the Effect of Flare and Ramp Angle on the Upstream Influence of Laminar and Transitional Reattaching Flows From Mach 3 to 7, AEDC TR-66-190, 1967.

11) Ginoux, J. J.: On Some Properties of Reattaching Laminar and Transitional High Speed Flows, VKI TN-53, 1969.

12) Needham, D. A. and Stollery, J. L.: Boundary Layer Separation in Hypersonic Flow, AIAA Paper 66-455, 1966.

13) Inger, G. R.: Scaling of Incipient Separation in High Speed Laminar Flows, AIAA Paper 93-3435, 1993.

14) Coleman, G. T. and Stollery, J. L.: Heat Transfer from Hypersonic Turbulent Flow at a Wedge Compression Corner, J. Fluid Mech., 56 (1972), pp. 741-752.

15) Elfstrom, G. M.: Turbulent Separation in Hypersonic Flow, IC Aero Report 71-16, 1971.

16) Coleman, G. T.: A Study of Hypersonic Boundary Layers Over a Family of Axisymmetric Bodies at Zero Incidence, IC Aero Report 73-06, 1973.

17) Stollery, J. L. and Bates, L.: Turbulent Hypersonic Viscous Interaction, J. Fluid Mech., 63 (1974), pp. 145-156.

18) Stollery, J. L., Maull, D. J. and Belcher, D. A.: The Imperial College Hypersonic Gun Tunnel, J. Royal Aero. Soc., 64 (1960), pp. 634-641.

19) Needham, D. A.: Progress Report on the Imperial College Hypersonic Gun Tunnel, IC Aero TN-118, 1963.

20) Kontis, K. and Stollery, J. L.: Control Effectiveness of a Jet-Slender Body Combination at Hypersonic Speeds, J. Spacecraft Rockets, 34 (1997), pp. 762-768.

21) Kontis, K.: Projectile Aerodynamics: Measurement and Computation, PhD. Thesis, Cranfield University, 1997.

22) Opatowski, T.: An Experimental Study of the Flow around and the 
Forces Developed by Hypersonic Lifting Vehicles, PhD. Thesis, University of London, 1967.

23) Coleman, G. T.: Hypersonic Boundary Layer Studies, PhD. Thesis, University of London, 1973.

24) Kontis, K., Qin, N., Stollery, J. L. and Edwards, J. A.: Hypersonic Performance of a Lifting Elliptic Cone with and without Strakes, $J$. Spacecraft Rockets, 37 (2000), pp. 21-28.

25) Kontis, K.: Surface Heat Transfer Measurements Inside a Supersonic Combustor by Laser Induced Fluorescence, J. Thermophys. Heat Transfer, 17 (2003), pp. 359-364.

26) Baldwin, B. S. and Lomax, H.: Thin Layer Approximation and Algebraic Model for Separated Turbulent Flows, AIAA Paper 78-0257, 1978.

27) Yee, H. C.: A Class of High Resolution Explicit and Implicit Shock Capturing Methods, NASA TM-101088, Oct. 1989.

28) Eiseman, P. R.: Grid Generation for Fluid Mechanics Computation, Annu. Rev. Fluid Mech., 17 (1985), pp. 487-522.

29) Kuehn, D. M.: Experimental Investigation of the Pressure Rise Required for the Incipient Separation of Turbulent Boundary in Two-Dimentional Supersonic Flow, NACA Memorandum 1-21-59A, 1958.

30) Drugge, G.: An Experimental Investigation of the Influence of Strong
Adverse Pressure Gradients on Turbulent Boundary Layers at Supersonic Speeds, FFA Report 47, 1953.

31) Holden, M. S.: Experimental Studies of Shock Wave Boundary Layer Interaction, VKI-LS-62, 1974.

32) Kessler, W. C., Reilly, J. F. and Mockapetris, L. J.: Supersonic Turbulent Boundary Layer Interaction with an Expansion Ramp and Compression Corner, Mc Donnell Douglas Report, MDC E0264, 1970.

33) Law, H. C.: Supersonic Turbulent Boundary Layer Separation Measurements at Reynolds Numbers of $10^{7}$ to $10^{8}$, AIAA Paper 73-665, 1973.

34) Sterrett, J. R. and Emery, J. C.: Experimental Separation Studies for Two-Dimensional Wedges and Curved Surfaces at Mach Numbers of 4.8 to 6.2, NASA TN D-1014, 1962.

35) Roshko, A. and Thomke, G. J.: Flare-Induced Interaction Lengths in Supersonic, Turbulent Boundary Layers, AIAA J., 14 (1974), pp. 873-879.

36) Appels, C.: Turbulent Boundary Layer Separation at Mach 12, VKI TN-90, 1973.

37) Simeonides, G., Hasse, W. and Manna, M.: Experimental, Analytical and Computational Methods Applied to Hypersonic Compression Ramp Flows, AIAA J., 32 (1994), pp. 189-195. 